\title{
Impulsividad y empatía en una muestra de delincuentes en tratamiento: diferencias entre delincuentes sexuales y no sexuales.
}

\section{Impulsivity and empathy in a sample of offenders under treatment: Differences between sexual and non-sexual offenders}

\section{resumen/alsstract:}

Tanto la impulsividad, entendida como rasgo de personalidad, como la empatía han sido consideradas como factores estrechamente relacionados con la delincuencia. El presente estudio evalúa la impulsividad y empatía en una muestra de delincuentes en tratamiento. Para ello, se analizaron los resultados obtenidos por 80 penados (74 varones y 6 mujeres) en el Cuestionario de personalidad $\mathrm{I7}$, cuyas escalas evalúan el nivel de irreflexión, temeridad y empatía de los sujetos evaluados. Las puntuaciones obtenidas por la muestra de delincuentes fueron comparables a las de muestras normativas. Sin embargo, al comparar los resultados obtenidos por el grupo de delincuentes sexuales con los del grupo de delincuentes no sexuales, se observaron puntuaciones significativamente más bajas en irreflexión y temeridad y un menor porcentaje de antecedentes penales en el grupo de delincuentes sexuales. Este estudio evidencia la necesidad de añadir instrumentos que midan la impulsividad en la evaluación de delincuentes en tratamiento, así como la inclusión de ingredientes terapéuticos específicos, que podrían incluir herramientas farmacológicas, en aquellos sujetos con un nivel más alto de impulsividad.

Both impulsivity, understood as a personality trait, and empathy have been considered as factors closely related to delinquency. The present study assesses impulsivity and empathy in a sample of offenders under treatment. To this end, the results obtained by 80 convicts (74 men and 6 women) in the 17 Personality Questionnaire 17 - which measures impulsiveness, venturesomeness and empathy- were analyzed. The scores obtained by the sample of offenders were comparable to those of normative samples. However, when comparing the results obtained by the group of sexual offenders with those of the group of non-sexual offenders, significantly lower scores were observed in impulsiveness and venturesomeness among sex offenders, as well as lower rates of recidivism and criminal records. This study evidences the need to add instruments that measure impulsivity in the assessment of offenders under treatment, as well as the inclusion of specific therapeutic ingredients, which could include pharmacological tools, in those subjects with higher levels of impulsivity.

\section{palabras clave/keywords:}

Delincuencia; personalidad; delincuentes sexuales; tratamiento; impulsividad; empatía.

Crime; personality; sex offenders; treatment; impulsivity; empathy. 


\section{Introducción}

Durante los últimos años, y con el fin principal de dar cumplimiento a las distintas responsabilidades penales alternativas, hemos presenciado un gran cambio en el abanico de programas de tratamiento para penados disponibles en España. Por un lado, en el año 2016 se produce por primera vez en Valencia la subdivisión del Programa Control de la Agresión Sexual (PCAS) (Rivera, Romero, Labrador, y Serrano, 2006), para penados que hayan cometido cualquier delito contra la libertad e indemnidad sexual, en dos programas específicos en función de la edad de las víctimas (adultas, PCAS-A; menores, PCAS-M). Por otro, contamos con nuevos programas de tratamiento dirigidos a otras tipologías delictivas, como Fuera de la Red (FDLR) (Herrero et al., 2015) -programa de intervención para delincuentes sexuales con víctimas menores de edad que hayan cometido sus delitos a través de las nuevas tecnologías (delitos de posesión y/o difusión de pornografía infantil, online child grooming, ...)-, el Programa de Intervención para Agresores de violencia de género en Medidas Alternativas (PRIA-MA) (Suárez et al., 2015) -destinado a hombres condenados por un delito de violencia de género-; PROSEVAL (Lijarcio et al., 2016) -programa de intervención psicoeducativa dirigido a personas condenadas por delitos contra la seguridad vial-; ENCUENTRO (Méndez et al., 2016) - programa de intervención en violencia familiar, destinado a cualquier persona adulta que haya ejercido algún tipo de violencia dentro de su entorno familiar (hacia sus padres, hijos, abuelos, nietos, pareja, etc.), exceptuando la violencia ejercida de los hombres hacia las mujeres (de género) - y, por último, PROBECO (Ballano, Suárez, Pérez, y Herrera, 2015) -programa de intervención para la sensibilización y reeducación en competencias sociales destinado a hombres y mujeres condenados/ as por diversos delitos (no previstos en los programas descritos anteriormente)-. Dichos programas se realizan en formato grupal e individual y van dirigidos a personas reclusas, ex-reclusas y sometidas a medidas penales alternativas a la prisión.

Es probable que un individuo que cometa un delito en una sociedad donde existen leyes reguladoras, respetadas por el resto de ciudadanos, presente dificultades para controlar sus impulsos y evaluar racionalmente las consecuencias de sus actos y, por tanto, actúe de acuerdo con estas malas evaluaciones (Baltieri y Boer, 2015). La impulsividad podría ser el reflejo de un estilo cognitivo característicamente impulsivo o incluso de un rasgo de personalidad (Arce y Santisteban, 2006), actuando como un factor de riesgo para diversos problemas conductuales como el consumo de drogas, la delincuencia o la agresividad (Arce y Santisteban, 2006; Baltieri y Boer, 2015). Por otro lado, los altos niveles de impulsividad se relacionan con un mayor riesgo de reincidencia (Baltieri y Boer, 2015); de hecho, una de las características de la impulsividad es la falta de sensibilidad al castigo (Arce y Santisteban, 2006). Si nos centramos en la delincuencia sexual, uno de los factores de riesgo para esta tipología delictiva es la presencia de parafilias, y más en concreto de pedofilia, desorden que podría tener un componente impulsivo. Diferentes estudios de neuroimagen indican que la pedofilia está asociada con alteraciones en la actividad y estructura del lóbulo frontal (Wiebking y Northoff, 2013), área central en la inhibición de la conducta. No obstante, parece que la impulsividad actúa como un factor de riesgo desinhibiendo la conducta, ya que los sujetos pedófilos que no habían delinquido mostraron un control inhibitorio superior mayor que los que sí que lo habían hecho (Kärgel et al., 2017). 
La impulsividad engloba múltiples componentes, incluyendo un comportamiento rápido, espontáneo, mal planificado, excesivo y potencialmente desadaptativo (Enticott y Ogloff, 2006), la falta de preocupación por las consecuencias, la preferencia por el refuerzo inmediato, el desprecio por las reglas, la propensión al aburrimiento, la falta de planificación, y la incapacidad para inhibir las respuestas (Baltieri y Boer, 2015). Según la teoría de Eysenck (Eysenck, Pearson, Easting, y Allsopp, 1985), todos esos componentes se agruparían en dos dimensiones distintas de la impulsividad (impulsivity): una correspondiente a la extraversión (temeridad, venturesomeness), y la otra más relacionada con el psicoticismo (irreflexión, impulsiveness). El neuroticismo, aunque ligeramente relacionado de forma positiva con la irreflexión, correlacionaría negativamente con la temeridad. De este modo, la irreflexión (hacer y decir cosas sin pensar, sin ser consciente del riesgo que conlleva) y la temeridad (buscar sensaciones, emociones y aventuras, siendo consciente del riesgo que implican), aunque relacionadas entre sí, estarían midiendo dos formas distintas de impulsividad.

Por otro lado, la literatura suele considerar la empatía -entendida como la capacidad de entender y sentir o simpatizar con el estado emocional de otro (Cohen y Strayer, 1996)-, como otro de los factores estrechamente relacionados con la delincuencia (Jolliffe y Farrington, 2004; Retz, Reif, Freitag, Retz-Junginger, y Rüsler, 2010). Siguiendo con el modelo de Eysenck (Eysenck et al., 1985), la empatía estaría relacionada positivamente con el neuroticismo, pero negativamente con el psicoticismo, y suele asociarse con el razonamiento moral, el comportamiento prosocial y la inhibición de la conducta antisocial (Jolliffe y Farrington, 2004). Sin embargo, otros estudios han revelado una relación débil entre la empatía y la agresión (verbal, física y sexual) (Vachon, Lynam, y Johnson, 2014), y la reincidencia sexual (Hanson y Morton-Bourgon, 2005); lo cual podría deberse a las dificultades para definirla y operativizarla correctamente (Barnett y Mann, 2013; Vachon et al., 2014). En el caso de la delincuencia sexual, los déficits de empatía podrían estar formando parte de los procesos cognitivos distorsionados que resultan en argumentos justificativos del delito (Marshall, Hamilton, y Fernandez, 2001). Por su parte, Barnett y Mann (2013) proponen un modelo de cinco componentes de la empatía en delincuentes sexuales (la toma de perspectiva, la capacidad de experimentar la emoción, la creencia de que los demás son dignos de compasión y respeto, factores situacionales y una capacidad para manejar el sufrimiento personal), en lugar del modelo tradicional de dos componentes (empatía cognitiva y emocional).

El presente estudio ex post facto tiene como objetivos los siguientes: 1) Evaluar la impulsividad y la empatía en una muestra de delincuentes en tratamiento 2) Identificar las diferencias entre los delincuentes sexuales y los no sexuales en estas variables; 3) Detectar las diferencias en función de diferentes variables criminológicas (v.g. reincidencia o presencia de antecedentes penales). En base estos objetivos, y a lo que señala la literatura previa, se establecen las siguientes hipótesis: a) La muestra de delincuentes obtendrá una puntuación mayor en impulsividad y menor en empatía que la población normal; b) Los delincuentes sexuales obtendrán puntuaciones más bajas en impulsividad y en empatía que los delincuentes no sexuales; c) Los delincuentes que tengan más antecedentes penales y hayan reincidido más obtendrán una puntuación mayor en impulsividad y menor en empatía. 
Los resultados del presente estudio pueden ser relevantes para comprender mejor el fenómeno criminal y las variables psicológicas que pueden explicarlo, y por lo tanto para prevenirlo mejor. Así mismo, es necesario conocer cómo influyen estas variables en el desarrollo de la conducta criminal para mejorar el diseño de los programas de tratamiento de delincuentes y para desarrollar mejores instrumentos de predicción de riesgo.

\section{Método}

\section{Participantes}

Los participantes del estudio fueron 80 penados (74 varones y 6 mujeres) por delitos de diversa índole, sometidos a programas de tratamiento en la Asociación Preven3 entre los años 2016 y 2017. El 65\% se encontraban en tratamiento en medio abierto por mandamiento judicial -por encontrarse en suspensión de condena (46.25\%), cumpliendo la pena de trabajos en beneficio de la comunidad (TBC) (16.25\%) o sometidos a una medida de libertad vigilada $(2.5 \%)$-, mientras que el $35 \%$ se había sometido voluntariamente a tratamiento psicológico en el Centro Penitenciario de Valencia “Antoni Asunción Hernández”. La edad media de los participantes fue de 42.21 años (Desviación Típica $(D T)=12.4$; rango: 21-78). El $82.5 \%$ eran españoles, seguidos por los de procedencia latino-americana $(11.25 \%)$ y los de países de Europa Oriental (3.75\%), un nacional británico y otro senegalés. El 30\% de la muestra contaba con antecedentes penales -la persona ya había sido condenada en firme en el pasado por la comisión de un delito- mientras que el $12.5 \%$ eran reincidentes -el delito por el que se les había condenado previamente estaba comprendido en el mismo Título del Código Penal y era de la misma naturaleza que el actual.

Los descriptivos de cada grupo de tratamiento pueden observarse en la Tabla 1.

Tabla 1. Descriptivos de cada grupo de tratamiento.

\begin{tabular}{lccccc}
\hline Programa & $\boldsymbol{n}(\%)$ & $\begin{array}{c}\text { Sexo } \\
{[n \text { hombres }(\%)]}\end{array}$ & $\begin{array}{c}\text { Edad } \\
M(D T)\end{array}$ & $\begin{array}{c}\text { Antece- } \\
\text { dentes } \\
\text { penales } \\
(\%)\end{array}$ & $\begin{array}{c}\text { Reinci- } \\
\text { dencia } \\
(\%)\end{array}$ \\
\hline PCAS-A & $8(10)$ & $8(100)$ & $49.63(10.84)$ & 50 & 12.5 \\
PCAS-M & $21(26.3)$ & $21(100)$ & $45.48(15.15)$ & 9.5 & 0 \\
FDLR & $14(17.5)$ & $14(100)$ & $42.57(12.36)$ & 21.4 & 14.3 \\
PRIA-MA* & $20(25)$ & $20(100)$ & $39.5(10.81)$ & 30 & 10 \\
PROBECO & $9(11.3)$ & $6(66.7)$ & $34.67(6.69)$ & 44.4 & 0 \\
PROSEVAL & $7(8.8)$ & $7(100)$ & $41.71(11.67)$ & 85.7 & 71.4 \\
ENCUENTRO & $3(3.8)$ & $0(0)$ & $40.33(4.04)$ & 0 & 0 \\
\hline
\end{tabular}

Nota. *Dos usuarios se encontraban simultáneamente en otro programa de tratamiento (PCAS-A y Probeco) cumpliendo con una pena de TBC. 
Tabla 1.(continuación). Descriptivos de cada grupo de tratamiento.

\begin{tabular}{lccccc}
\hline \multirow{5}{*}{ Programa } & \multicolumn{5}{c}{$\begin{array}{c}\text { Situación penal } \\
n(\%)\end{array}$} \\
\cline { 2 - 6 } & $\begin{array}{l}\text { Suspensión } \\
\text { de condena }\end{array}$ & TBC & Libertad vigilada & Prisión & Prisión provisional \\
\hline PCAS-A & $3(37.5)$ & $1(12.5)$ & 0 & $4(50)$ & 0 \\
PCAS-M & $10(47.6)$ & $1(4.8)$ & $1(4.8)$ & $10(42.9)$ & 0 \\
FDLR & $7(50)$ & 0 & 0 & $5(35.7)$ & $2(14.3)$ \\
PRIA-MA* & $11(55)$ & 0 & $1(5)$ & $8(40)$ & 0 \\
PROBECO & 0 & $9(100)$ & 0 & 0 & 0 \\
PROSEVAL & $3(42.9)$ & $4(57.1)$ & 0 & 0 & 0 \\
ENCUEN- & $3(100)$ & 0 & 0 & 0 & 0 \\
TRO & & 0 & 0 & 0 \\
\hline
\end{tabular}

Nota. *Dos usuarios se encontraban simultáneamente en otro programa de tratamiento (PCAS-A y Probeco) cumpliendo con una pena de TBC.

\section{Instrumento}

Para evaluar a la muestra, se utilizó la versión española del Cuestionario de personalidad I7 (Eysenck et al., 1985; Luengo, Carrillo-de-la-Peña, y Otero, 1991). Se trata de un cuestionario autoinformado que consta de 54 ítems binarios que evalúan tres rasgos secundarios de personalidad en el marco de la teoría de Eysenck: irreflexión (19 ítems), temeridad (16 ítems) y empatía (19 ítems).

La irreflexión, la temeridad y la empatía son tres factores robustos que pueden medirse de forma adecuada con el Cuestionario I7 (Eysenck et al., 1985). Anteriores estudios han evidenciado un alpha de Cronbach de .84 para la irreflexión, .83 para la temeridad y .67 para la empatía (Aluja y Blanch, 2007).

\section{Procedimiento}

Todos los usuarios de los programas de tratamiento $(N=102)$ pasaron por una fase de evaluación y motivación previa a las sesiones de intervención de los programas de tratamiento. Dentro de esta fase, y tras firmar un documento de consentimiento informado, respondieron individualmente al cuestionario I7. Aquellos cuestionarios con más de un ítem no respondido $(n=6)$ no fueron incluidos en los análisis. Por otro lado, 16 usuarios no llegaron a completar el cuestionario por abandono o expulsión de sus respectivos programas. El número total de cuestionarios analizados fue de $n=80$.

Los datos sociodemográficos y judiciales fueron extraídos de la documentación facilitada por el Servicio de Gestión de Penas y Medidas Alternativas (SGPMA) y el Centro Penitenciario de Valencia, y posteriormente codificados de forma anónima. 
Tabla 2. Puntuaciones obtenidas en el cuestionario I7 por nuestra muestra de delincuentes en tratamiento y por una muestra de estudiantes universitarios.

\begin{tabular}{ccccc}
\hline & \multicolumn{2}{c}{$\begin{array}{c}\text { Presente estudio } \\
(N=80)\end{array}$} & \multicolumn{2}{c}{$\begin{array}{c}\text { Luengo et al. (1991) } \\
(N=307)\end{array}$} \\
\cline { 2 - 5 } I7 & $M$ & DT & $M$ & $D T$ \\
\hline Irreflexión & 5.66 & 4.69 & 6.64 & 3.99 \\
Temeridad & 6.3 & 3.26 & 9.6 & 3.83 \\
Empatía & 12.49 & 2.62 & 14.46 & 2.78 \\
\hline
\end{tabular}

\section{Análisis de datos}

En primer lugar, se analizaron los resultados obtenidos por toda la muestra en el cuestionario 17 en las escalas de irreflexión, temeridad y empatía. Estos se compararon con los obtenidos por Luengo et al. (Luengo et al., 1991) en una muestra de estudiantes universitarios, aunque no se pudieron comparar ambas muestras usando pruebas estadísticas por no poder acceder a los datos completos de la distribución. Asimismo, se calculó la consistencia interna del cuestionario para nuestra muestra mediante el alpha de Cronbach,

En segundo lugar, se compararon los resultados obtenidos por aquellos usuarios que participaron en los programas de tratamiento para delincuentes sexuales ${ }^{1}$ (programas PCAS-A, PCAS-M y FDLR) con los del resto de la muestra; dado que el tamaño de cada uno de los grupos de tratamiento por separado no permitía realizar contrastes con la suficiente potencia estadística. Para ello, y debido al incumplimiento de los supuestos de normalidad de los datos para las tres variables (irreflexión, temeridad y empatía) según el test de KolmogorovSmirnov $(p<.05)$, se llevó a cabo la prueba U de Mann-Whitney para el contraste de medias entre dos grupos independientes. Asimismo, se llevaron a cabo pruebas Ji-cuadrado para la comparación de los datos categóricos entre ambos grupos. Se utilizó una prueba $t$ de Student para muestras independientes para comparar la edad de ambos grupos, dado que esta variable sí se distribuía normalmente $(K-S(80)=.062 ; p=.2)$.

En tercer lugar, se utilizó la prueba U de Mann-Whitney para comparar los resultados obtenidos por aquellos penados que contaban con antecedentes penales frente a los de los delincuentes primarios, así como los de los reincidentes frente a los no reincidentes.

Todos los análisis fueron realizados con el paquete estadístico IBM SPSS Statistics (versión 23).

\footnotetext{
1 El usuario que se encontraba realizando simultáneamente el programa PRIA-MA (prioritario) y el PCAS-A (complementario) no fue incluido dentro del grupo de delincuentes sexuales para no sesgar el estudio. El delito cometido se incardinaba dentro del ámbito de la pareja y bajo un clima de machismo.
} 


\section{Resultados}

\section{Irreflexión, temeridad y empatía en delincuentes en tratamiento}

Pueden observarse en la Tabla 2 las puntuaciones obtenidas por la muestra de delincuentes en tratamiento $(N=80)$ en las escalas Irreflexión, Temeridad y Empatía, junto con las obtenidas por una muestra de estudiantes universitarios $(N=307)$ (Luengo et al., 1991). Las medias en las tres variables son ligeramente inferiores en la muestra de delincuentes en tratamiento. La consistencia interna (alpha de Cronbach) del cuestionario I7 en la presente muestra fue de .87 para la irreflexión, .7 para la temeridad y .49 para la empatía.

Tabla 3. Puntuación media y desviación típica obtenida en el cuestionario I7 por cada grupo de tratamiento.

\begin{tabular}{ccccc}
\hline Programa & $\boldsymbol{n}$ & $\begin{array}{c}\text { Irreflexión } \\
M(D T)\end{array}$ & $\begin{array}{c}\text { Temeridad } \\
M(D T)\end{array}$ & $\begin{array}{c}\text { Empatía } \\
M(D T)\end{array}$ \\
\hline PCAS-A & 8 & $3.38(2.88)$ & $4.75(3.01)$ & $11.38(3.29)$ \\
PCAS-M & 21 & $5.48(5.34)$ & $5.29(3.04)$ & $13.29(2.28)$ \\
FDLR & 14 & $5.14(5.46)$ & $6.14(3.18)$ & $13(2.69)$ \\
PRIA-MA* & 20 & $7.3(4.58)$ & $8.9(2.63)$ & $12.7(2.36)$ \\
PROBECO & 9 & $5.11(2.98)$ & $5.44(2.88)$ & $11.67(2.5)$ \\
PROSEVAL & 7 & $6.71(3.55)$ & $5(2.31)$ & $10.71(2.14)$ \\
ENCUENTRO & 3 & $4.67(6.35)$ & $8.33(4.62)$ & $13.33(4.04)$ \\
\hline
\end{tabular}

Nota. *Dos usuarios se encontraban simultáneamente en otro programa de tratamiento (PCAS-A y Probeco) cumpliendo con una pena de TBC.

\section{Delincuentes sexuales frente a otros delincuentes}

Las puntuaciones obtenidas por ambos grupos pueden observarse en la Tabla 4. El grupo de delincuentes sexuales puntuó significativamente más bajo en Irreflexión $(p=.032)$ y Temeridad $(p=.01)$, no existiendo diferencias significativas en la escala de Empatía $(p=.16)$.

Tabla 4. Puntuación media y desviación típica obtenida en el cuestionario I7 por delincuentes sexuales y no sexuales.

\section{Delincuentes sexuales Delincuentes no sexuales}

\begin{tabular}{ccccccc}
\multicolumn{2}{c}{$(n=42)$} & \multicolumn{2}{c}{$(n=38)$} \\
\hline $\mathbf{I 7}$ & $M$ & $D T$ & $M$ & $D T$ & $U$ & $p$ \\
\hline Irreflexión & 4.86 & 4.98 & 6.55 & 4.22 & 577 & $\mathbf{. 0 3 2}$ \\
\hline Temeridad & 5.43 & 3.08 & 7.26 & 3.21 & 531 & $\mathbf{. 0 1 0}$ \\
\hline Empatía & 12.79 & 2.66 & 12.16 & 2.56 & 944 & .156
\end{tabular}

Nota. Los valores en negrita son estadísticamente significativos $(p<.05)$. 
La prueba de Ji-cuadrado evidenció que el grupo de delincuentes sexuales contaba con un menor porcentaje de antecedentes penales, aunque no se hallaron diferencias estadísticamente significativas en sus tasas de reincidencia (ver Tabla 5). Por otro lado, el grupo de delincuentes sexuales mostró una edad significativamente más alta (Delincuentes sexuales: media $=45.29 ; D T=13.66$. Delincuentes no sexuales: media $=38.82 ; D T=9.94 . t(74.69)$ $=2.44 ; p=.017)$.

Tabla 5. Diferencias entre delincuentes sexuales y no sexuales en variables criminológicas.

\section{Delincuentes sexuales Delincuentes no sexuales}

\begin{tabular}{cccc}
$(n=42)$ & $(n=38)$ & \\
\hline$n(\%)$ & $n(\%)$ & $\chi^{\wedge} 2^{*}$ & $p$
\end{tabular}

Variable

\begin{tabular}{lccrr}
\hline Antecedentes penales & $8(19)$ & $16(42.1)$ & 5.05 & $\mathbf{. 0 2 5}$ \\
\hline Reincidencia & $3(7.1)$ & $7(18.4)$ & 1.4 & .236 \\
\hline
\end{tabular}

Nota. Los valores en negrita son estadísticamente significativos $(\mathrm{p}<.05)$.

*Se utilizó la corrección de Yates cuando al menos una casilla de la tabla tenía un recuento esperado menor de 5 .

\section{Primariedad delictiva y reincidencia}

Las puntuaciones obtenidas por sujetos primarios delictivamente y con antecedentes penales, no reincidentes y reincidentes pueden observarse en la Tabla 6. Aquellos con antecedentes penales puntuaron significativamente más alto en la escala de Irreflexión ( $p=$ .032), no existiendo diferencias significativas en las escalas de Temeridad ( $p=.74$ ) y Empatía $(p=.824)$. No se encontraron diferencias estadísticamente significativas entre los reincidentes y los no reincidentes.

Tabla 6. Puntuación media y desviación típica obtenida en el cuestionario I7 por delincuentes primarios y con antecedentes penales, no reincidentes y reincidentes.

\begin{tabular}{ccccccccccccc}
\hline & $\begin{array}{c}\text { Sin anteceden- } \\
\text { tes penales } \\
(n=56)\end{array}$ & $\begin{array}{c}\text { Con antece- } \\
\text { dentes penales } \\
(n=24)\end{array}$ & & \multicolumn{3}{c}{$\begin{array}{c}\text { No reinci- } \\
\text { dentes } \\
(n=70)\end{array}$} & \multicolumn{2}{c}{$\begin{array}{c}\text { Reinciden- } \\
\text { tes } \\
(n=10)\end{array}$} \\
\hline I7 & $M$ & $D T$ & $M$ & $D T$ & $U$ & $p$ & $M$ & $D T$ & $M$ & $D T$ & $U$ & $p$ \\
\hline Irreflexión & 5.04 & 4.72 & 7.13 & 4.35 & 874.5 & $\mathbf{. 0 3 2}$ & 5.44 & 4.57 & 7.2 & 5.43 & 423 & .285 \\
\hline Temeridad & 6.25 & 3.18 & 6.42 & 3.49 & 703.5 & .74 & 6.17 & 3.22 & 7.2 & 3.52 & 410.5 & .376 \\
\hline Empatía & 12.52 & 2.65 & 12.42 & 2.6 & 651 & .824 & 12.5 & 2.66 & 12.4 & 2.41 & 340 & .883 \\
\hline
\end{tabular}

Nota. Los valores en negrita son estadísticamente significativos $(p<.05)$. 


\section{Discusión}

El presente estudio es el primero en analizar las puntuaciones obtenidas en el cuestionario I7 en una muestra de delincuentes en tratamiento en la Comunidad Valenciana. Asimismo, es el primero que compara los resultados obtenidos por delincuentes sexuales y no sexuales en este instrumento en España. Por ello, los resultados no pueden ser comparados al detalle con los de otras muestras similares, aunque sí parecen consistentes con algunas investigaciones previas.

En cuanto al primer objetivo del estudio, los resultados indican que la muestra obtiene medias en las tres escalas que mide el cuestionario (5.66 de un máximo de 19 en Irreflexión; 6.3 de 16 en Temeridad y 12.49 de 19 en Empatía). Estas puntuaciones parecen ligeramente inferiores a las obtenidas en muestras normativas como la de Luengo et al. (1991), lo cual contrastaría con lo observado en estudios previos (Arce y Santisteban, 2006; Baltieri y Boer, 2015) y con la primera hipótesis expuesta. Sin embargo, al centrarnos en cada programa de tratamiento por separado, podemos observar que aquellos usuarios del programa PRIA-MA obtienen las puntuaciones más altas en las dos escalas relacionadas con la impulsividad, puntuaciones apreciablemente superiores a las obtenidas por Pérez, Giménez-Salinas y de Juan (2010). Esto podría deberse a que nuestra muestra incluía penados por delitos de violencia de género en medio cerrado, cumpliendo penas privativas de libertad de larga duración, habitualmente asociadas a hechos más graves y violentos que los cometidos por los usuarios de los programas en medio abierto. Los usuarios con los niveles de empatía más bajos fueron los del programa PROSEVAL, algo sorprendente teniendo en cuenta que se trata de penados por delitos contra la seguridad vial -sin víctimas concretas identificables-, aunque congruente con la escasa correlación observada entre la empatía y la agresión en el estudio de Vachon et al. (2014). Por otro lado, la pobre consistencia interna observada en la escala de empatía $(\alpha=.49)$ en la presente muestra impide llegar a conclusiones fiables sobre las puntuaciones obtenidas en la misma.

En cuanto al segundo objetivo del presente estudio, el grupo de delincuentes sexuales puntuó significativamente más bajo en las escalas de Impulsividad que el resto de delincuentes, lo cual apoyaría parcialmente la segunda hipótesis planteada. Sin embargo, su edad también era significativamente más alta. En este sentido, la literatura evidencia un descenso en los niveles de irreflexión y temeridad con la edad (Crews y Boettiger, 2009; Eysenck et al., 1985), mientras que los niveles de empatía se mantienen constantes a lo largo de los años (Eysenck et al., 1985). Por otro lado, diferentes estudios indican que los delincuentes sexuales constituyen una población heterogénea y que no existe un perfil de personalidad prototípico, característico de este colectivo (Davis y Archer, 2010; Perrot, Bénony, Chahraoui, y Juif, 2014). En nuestro estudio, la muestra de delincuentes sexuales contaba con una amplia mayoría de usuarios en los programas para delitos sexuales contra menores de edad (34 frente a 8). Este colectivo no suele diferenciarse significativamente de la población normal en sus niveles de impulsividad (Cohen y Galynker, 2002), mientras que aquellos delincuentes sexuales con víctimas adultas sí se caracterizan por la impulsividad en su modus operandi (Cohen, Frenda, Mojtabai, Katsavdakis, y Galynker, 2007) -especialmente los agresores sexuales seriales (Baltieri y Andrade, 2008). Asimismo, se observa una mayor 
proporción de delincuentes primarios en el grupo de delincuentes sexuales, lo que sería congruente con sus bajas puntuaciones en las escalas de impulsividad: son conscientes del riesgo que conllevan sus actos y prefieren evitarlos, pensando antes de actuar. Por otro lado, esto podría indicar que las motivaciones delictivas de la muestra de delincuentes sexuales incluida en este estudio fueran más parafílicas o sexualizadas, lo cual está asociado con una menor impulsividad (Baltieri y Boer, 2015; Eastvold et al., 2011).

Respecto al tercer objetivo, aquellos usuarios con un historial delictivo previo mostraban mayores niveles de irreflexión que los delincuentes primarios, lo cual apoyaría la tercera de las hipótesis. Sin embargo, el reducido número de delincuentes reincidentes $(n=10)$ frente a los no reincidentes $(n=70)$ impedía realizar contrastes potentes entre ambos grupos. La irreflexión puede distinguirse del otro componente de la impulsividad, la temeridad, en que el sujeto no es consciente del riesgo que conllevan sus actos, actuando sin reparar en ellos (Eysenck et al., 1985; Luengo et al., 1991); lo que nos llevaría a pensar que sus esquemas cognitivos y teorías implícitas podrían tener un importante papel en aquellos sujetos con antecedentes penales. Las teorías implícitas afectan a la capacidad de predicción de la conducta de los otros, la elección de los propios actos y las expectativas sobre la reacción de los otros (Szumski y Zielona-Jenek, 2016). Por ejemplo, la teoría implícita de un delincuente sexual podría construirse en torno a la creencia de que la actividad sexual de un adulto con un niño no es perjudicial para el menor, que es neutral o incluso gratificante para él/ella; una distorsión cognitiva que legitimaría la conducta delictiva (Ward y Keenan, 1999).

Nuestros hallazgos podrían tener implicaciones para el manejo terapéutico diferencial de los distintos subgrupos de delincuentes. La literatura muestra que los sistemas dopaminérgicos y serotoninérgicos pueden estar a la base de la conducta impulsiva (Schilling, Kühn, Sander, y Gallinat, 2014). En este sentido, los delincuentes más impulsivos (delincuentes no sexuales y penados por violencia de género) podrían beneficiarse de intervenciones psicológicas en combinación con tratamientos farmacológicos (v.gr. fármacos inhibidores selectivos de la recaptación de la dopamina y/o la serotonina) y siempre tras una evaluación psiquiátrica individualizada. Teniendo en cuenta que la impulsividad, entendida como el bajo auto-control, es un concepto central en la explicación contemporánea de la delincuencia (Hirschi, 2004), sería fundamental incluir instrumentos que la midan en el protocolo de evaluación previo a la aplicación de un programa de tratamiento para la prevención de la delincuencia, para un mejor ajuste de la intervención atendiendo a esta variable. Por otro lado, podría resultar de interés incluir ingredientes terapéuticos dirigidos a mejorar las estrategias de auto-control emocional y conductual -como ya se hace en los programas PRIAMA (Suárez et al., 2015), PROBECO (Ballano et al., 2015) y PROSEVAL (Lijarcio et al., 2016) - con aquellos colectivos que presenten una tendencia impulsiva más prominente. Con respecto a la empatía, no se han apreciado déficits considerables en nuestra muestra, y, sin embargo, muchos programas de tratamiento -especialmente aquellos dirigidos a delincuentes sexuales (Herrero et al., 2015; Rivera et al., 2006)- dedican una gran parte de sus contenidos a la empatía con la víctima, a pesar de que la evidencia empírica de su utilidad es pobre y no existe un modelo teórico coherente que explique cómo podría contribuir al cambio (Mann y Barnett, 2012). 
No obstante, los hallazgos encontrados en el presente estudio presentan algunas limitaciones que es necesario señalar. En primer lugar, el pequeño tamaño de cada uno de los grupos de tratamiento reduce significativamente la potencia estadística de los cálculos y por tanto hace que los resultados tengan que ser analizados con cautela. En segundo lugar, la heterogeneidad de algunos de los grupos de tratamiento en cuanto al tipo de delito (v.g. PROBE$\mathrm{CO}$ ) dificulta la generalización de los resultados. En tercer lugar, la diferencia en la edad de los diferentes grupos podría, en parte, explicar las diferencias entre delincuentes sexuales y no sexuales, al tratarse de una variable moderadora de la conducta impulsiva. Por último, la comparación de los resultados con los obtenidos por población normativa está limitada por carecer de los datos de la distribución completa, lo que no permitió utilizar herramientas estadísticas más potentes.

En cuanto a la investigación futura, sería necesario utilizar muestras más grandes, que permitieran utilizar estadística paramétrica y así elevar la potencia de los cálculos y, por tanto, la fiabilidad de los resultados. También sería interesante contar con muestras mayores de delincuentes sexuales para poder realizar comparaciones en función de la edad de la víctima, la presencia o ausencia de parafilias, o la relación con la víctima, y así poder ajustar mejor los tratamientos en función del perfil del delincuente. Asimismo, será necesario en el futuro relacionar esta variable con otras relevantes para la explicación del fenómeno criminal (v.g. trastornos de la personalidad, emocionales o de la conducta, así como condiciones neurobiológicas concretas, la presencia de distorsiones cognitivas o de otros factores de riesgo), para poder conocer el poder predictivo de estas variables o la correlación existente entre ellas.

"Los autores agradecen la colaboración en este estudio de los terapeutas de la Asociación Preven3, así como a la Secretaría General de Instituciones Penitenciarias por autorizar esta investigación. Este trabajo ha sido realizado parcialmente con el apoyo de una beca FPU, financiada por el Ministerio de Educación, Cultura y Deporte."

\section{Referencias}

Aluja, A., y Blanch, A. (2007). Comparison of impulsiveness, venturesomeness and empathy (I7) structure in English and Spanish samples: Analysis of different structural equation models. Personality and Individual Differences, 43, 2294-2305. doi:10.1016/j.paid.2007.07.019

Arce, E., y Santisteban, C. (2006). Impulsivity: a review. Psicothema, 18, 213-220.

Ballano, E., Suárez, A., Pérez, S., y Herrera, M. J. (2015). PROBECO: Programa de intervención, sensibilización y reeducación en competencias sociales. Madrid: Ministerio del Interior. Secretaría General Técnica.

Baltieri, D. A., y Andrade, A. G. (2008). Comparing serial and nonserial sexual offenders: Alcohol and street drug consumption, impulsiveness and history of sexual abuse. Revista Brasileira de Psiquiatria, 30(1), 25-31.

Baltieri, D. A., y Boer, D. P. (2015). Two clusters of child molesters based on impulsiveness. Revista Brasileira de Psiquiatria, 37, 139-145. doi:10.1590/1516-4446-2014-1568

Barnett, G. D., y Mann, R. E. (2013). Cognition, Empathy, and Sexual Offending. Trauma, Violence, \& Abuse, 14(1), 22-33. doi:10.1177/1524838012467857

Cohen, D., y Strayer, J. (1996). Empathy in conduct-disordered and comparison. Developmental Psychology, 32(6), 988998. 
Cohen, L. J., y Galynker, I. (2002). Clinical features of pedophilia and implications for treatment. Journal of Psychiatric Practice, 8, 1-14.

Cohen, L. J., Frenda, S., Mojtabai, R., Katsavdakis, K., y Galynker, I. (2007). Comparison of sexual offenders against children with sexual offenders against adolescents and adults: Data from the New York State Sex Offender Registry. Journal of Psychiatric Practice, 13(6), 373-384.

Crews, F. T., y Boettiger, C. A. (2009). Impulsivity, frontal lobes and risk for addiction. Pharmacology Biochemistry and Behavior, 93, 237-247.

Davis, K. M., y Archer, R. P. (2010). A critical review of objective personality inventories with sex offenders. Journal of Clinical Psychology, 66(12), 1254-1280. doi:10.1002/jclp.20722

Eastvold, A., Suchy, Y., y Strassberg, D. (2011). Executive function profiles of pedophilic and nonpedophilic child molesters. Journal of the International Neuropsychological Society, 17, 295-307.

Enticott, P. G., y Ogloff, J. R. P. (2006). Elucidation of impulsivity. Australian Psychologist, 41, 3-14. doi: $10.1080 / 00050060500391894$

Eysenck, S. B., Pearson, P. R., Easting, G., y Allsopp, J. F. (1985). Age norms for impulsiveness, venturesomeness and empathy in adults. Personality and Individual Differences, 6(5), 613-619.

Hanson, R. K., y Morton-Bourgon, K. E. (2005). The characteristics of persistent sexual offenders: A meta-analysis of recidivism studies. Journal of Consulting and Clinical Psychology, 73, 1154-1163.

Herrero, 0., Negredo, L., Lila, M., García, A., Pedrón, V., y Terreros, E. (2015). Fuera de la Red: programa de intervención frente a la delincuencia sexual con menores en la Red. Madrid: Ministerio del Interior. Secretaría General Técnica.

Hirschi, T. (2004) Self-control and crime. En R. F. Baumeister, y K. D. Vohs (Eds), Handbook of Self-regulation: Research, Theory, and Applications (pp. 537-552). New York: The Guilford Press.

Jolliffe, D., y Farrington, D. P. (2004). Empathy and offending: A systematic review and meta-analysis. Aggression and Violent Behavior, 9, 441-476. doi:10.1016/j.avb.2003.03.001

Kärgel, C., Massau, C., Weiß, S., Walter, M., Borchardt, V., Krueger, T. H., .. Schiffer, B. (2017). Evidence for superior neurobiological and behavioral inhibitory control abilities in non-offending as compared to offending pedophiles. Human Brain Mapping, 38(2), 1092-1104. doi:10.1002/hbm.23443

Lijarcio, J. I., Escamilla, C., López, C., Puchades, R., Martí-Belda, A., Bosó, P., y Montoro, L. (2016). PROSEVAL: Programa de Intervención psicoeducativa en seguridad vial. Manual para el profesional. Madrid: Ministerio del Interior. Secretaría General Técnica.

Luengo, M. A., Carrillo-de-la-Peña, M. T., y Otero, J. M. (1991). The components of impulsiveness: A comparison of the I.7 impulsiveness questionnaire and the Barratt impulsiveness scale. Personality and Individual Differences, 12(7), 657-667.

Mann, R. E., y Barnett, G. D. (2012). Victim empathy intervention with sexual offenders: rehabilitation, punishment or correctional quackery? Sexual Abuse, 25(3), 282-301. doi:10.1177/1079063212455669

Marshall, W. L., Hamilton, K., y Fernandez, Y. M. (2001). Empathy deficits and cognitive distortions in child molesters. Sexual Abuse, 13(2), 123-130.

Méndez, R. C., Suárez, A., Negredo, L., Rodrigo, M. J., Lobo, M., Pérez, S., . . Álvarez, G. (2016). Programa ENCUENTR0: Programa de intervención drente a la violencia familiar en medidas alternativas. Manual para el profesional. Madrid: Ministerio del Interior. Secretaría General Técnica.

Pérez, M., Giménez-Salinas, A., y de Juan, M. (2010). Evaluación del programa “Violencia de género: Programa de intervención para agresores", en medidas alternativas. Madrid: Ministerio del Interior. Secretaría General Técnica.

Perrot, M., Bénony, H., Chahraoui, K., y Juif, C. (2014). Étude des tendances dysfonctionnelles de la personnalité chez des auteurs d'infractions sexuelles. L'Encéphale, 40(5), 366-372. doi:10.1016/j.encep.2014.01.003

Retz, W., Reif, A., Freitag, C. M., Retz-Junginger, P., y Rüsler, M. (2010). Association of a functional variant of neuronal nitric oxide synthase gene with self-reported impulsiveness, venturesomeness and empathy in male offenders. Journal of Neural Transmission, 117, 321-324. doi:10.1007/s00702-009-0352-4 
Rivera, G., Romero, M. C., Labrador, M. A., y Serrano, J. (2006). El control de la agresión sexual: programa de intervención en el medio penitenciario. Manual del terapeuta. Madrid: Ministerio del Interior. Secretaría General Técnica.

Schilling, C., Kühn, S., Sander, T., y Gallinat, J. (2014). Association between dopamine D4 receptor genotype and trait impulsiveness. Psychiatric Genetics, 24(2), 82. doi:10.1097/ypg.0000000000000005

Stein, D. J., Black, D. W., y Pienaar, W. (2000). Sexual disorders not otherwise specified: Compulsive, addictive, or impulsive? CNS Spectrums, 5, 60-64.

Suárez, A., Méndez, R., Negredo, L., Fernández, M. N., Muñoz, J. M., Carbajosa, P., . . Herrero, 0. (2015). Programa de intervención para agresores de violencia de género en medidas alternativas (PRIA-MA). Manual para el profesional. Madrid: Ministerio del Interior. Secretaría General Técnica.

Szumski, F., y Zielona-Jenek, M. (2016). Child molesters' cognitive distortions. Conceptualizations of the term. Psychiatria Polska, 50(5), 1053-1063. doi:10.12740/PP/37470

Vachon, D. D., Lynam, D. R., y Johnson, J. A. (2014). The (Non)Relation Between Empathy and Aggression: Surprising Results. Psychological Bulletin, 140(3), 751-773. doi:10.1037/a0035236

Ward, T., y Keenan, T. (1999). Child molesters' implicit theories. Journal of Interpersonal Violence, 14(8), 821-838.

Wiebking, C., y Northoff, G. (2013). Neuroimaging in pedophilia. Current Psychiatric Reports, 15(4), 351-360. doi:10.1007/ s11920-013-0351-x 